\title{
STUDY OF HADRONIC HYDROGEN-LIKE ATOMS IN THE DIRAC EXPERIMENT AT PS CERN*
}

\author{
LEONID AfAnASyeV \\ for the DIRAC Collaboration \\ Joint Institute for Nuclear Research, Dubna, Moscow region, Russia \\ (Received August 21, 2017) \\ Production of hadronic hydrogen-like atoms at high-energy collisions \\ and method of their observation are considered. Main results of the DIRAC \\ experiment on observation and lifetime measurement of atoms formed by \\ pairs of charged pion-pion and pion-kaon are presented.
}

DOI:10.5506/APhysPolB.48.1889

\section{Physics of hadronic hydrogen-like atoms}

The DIRAC experiment aims to observe and study hydrogen-like atoms formed by pairs of $\pi^{+} \pi^{-}\left(A_{2 \pi}\right)$ and $\pi^{ \pm} K^{\mp}\left(A_{\pi K}\right)$ mesons using the $24 \mathrm{GeV}$ extracted beam of PS CERN. The lifetime of these atoms is dictated by the strong interaction between the components. Thus combining hadrons into a hydrogen-like atom opens a unique possibility to study a property of the strong interaction at the very low relative momenta which are of the order of the atom Bohr momentum. For $\pi^{+} \pi^{-}$atom it is $0.5 \mathrm{MeV}$, for $\pi K$ - $0.8 \mathrm{MeV}$. Hence, the region of QCD confinement becomes available for investigation.

The ground-state lifetime of $\pi^{+} \pi^{-}$atom $\tau_{2 \pi}$ is governed by the $\pi \pi$ $S$-wave scattering lengths $a_{I}$, with isospin $I=0,2[1-3]: 1 / \tau_{2 \pi} \propto\left|a_{0}-a_{2}\right|^{2}$. For the $\pi K$ atom, the lifetime $\tau_{\pi K}$ depends on the $\pi K S$-wave scattering lengths with isospin $1 / 2$ and $3 / 2$ [4]: $1 / \tau_{\pi K} \propto\left|a_{1 / 2}-a_{3 / 2}\right|^{2}$. The values of these scattering lengths can be rigorously calculated in the Chiral Perturbation Theory (ChPT) [5, 6]. Last results of Lattice QCD [7] confirm this calculation. Thus, the measurement of the hadronic hydrogen-like atom lifetimes provides an experimental test of the low-energy QCD predictions.

* Presented at the $2^{\text {nd }}$ Jagiellonian Symposium on Fundamental and Applied Subatomic Physics, Kraków, Poland, June 3-11, 2017. 
In collisions with target nuclei, high-energy protons can produce pairs of oppositely charged mesons. The final-state Coulomb interaction leads to an enhancement in production of such pairs at low relative c.m. momentum $Q$ ("Coulomb pairs") and to the formation of bound states or hydrogen-like atoms. The production cross sections of the free and bound states are related as the square of Coulomb wave functions for these states. Moving after the production in the target, the $\pi^{+} \pi^{-}\left(\pi^{ \pm} K^{\mp}\right)$ atoms may either decay into $\pi^{0} \pi^{0}\left(\pi^{0} K^{0}, \pi^{0} \bar{K}^{0}\right)$ or evolve by excitation (de-excitation) to different quantum states and, finally, decay or survive (long-lived states) or break up (be ionized) by the electric field of the target atoms [8]. In the case of breakup, characteristic "atomic pairs" emerge with a low relative momentum $Q$ in their center of mass $(Q<3 \mathrm{MeV} / c)$, and small opening angle in the laboratory frame $(<3 \mathrm{mrad})$. These pairs are the subject of observation. A numerical description of atom passage through the target allows to obtain a relation between the probability of atom breakup and the atom lifetime for decay.

A high-resolution magnetic spectrometer $\left(\Delta p / p \sim 3 \times 10^{-3}\right)$ is used [9] (Fig. 1) to identify the pairs and measure the relative momentum $Q$ with a sufficient precision to detect the "atomic pairs". This signal lies above

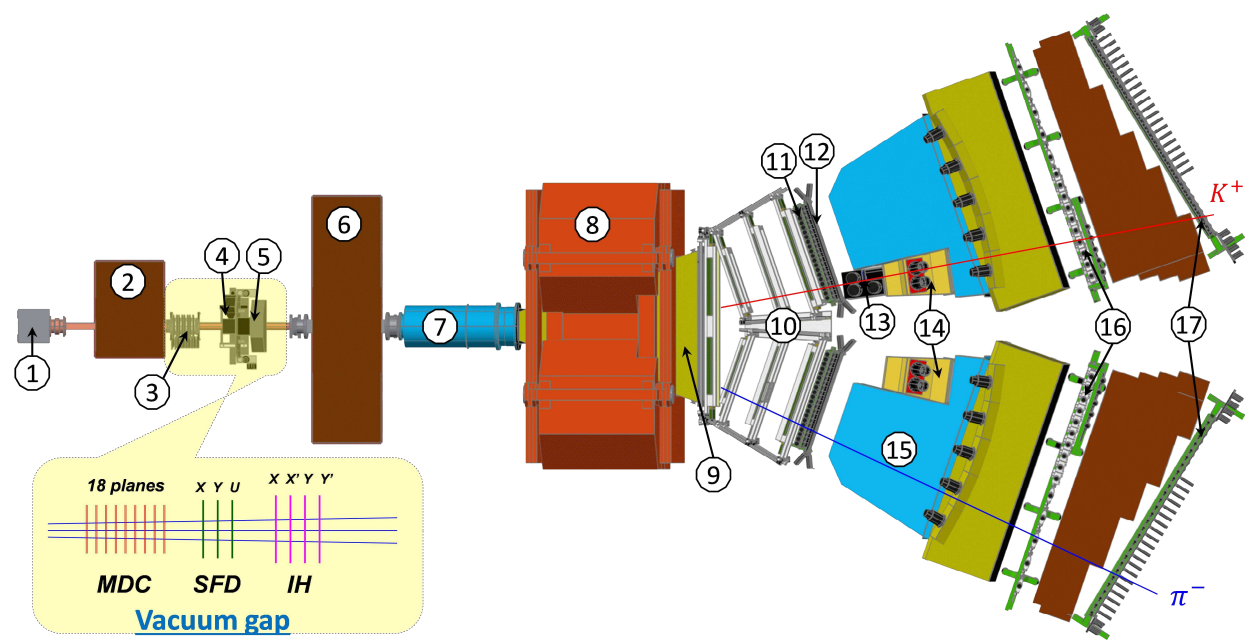

Fig. 1. General view of the DIRAC setup (1 - target station; 2 - first shielding; 3 - micro drift chambers (MDC); 4 - scintillating fiber detector (SFD); 5 - ionization hodoscope $(\mathrm{IH}) ; 6$ - second shielding; 7 - vacuum tube; 8 spectrometer magnet; 9 - vacuum chamber; 10 - drift chambers (DC); 11 - vertical hodoscope $(\mathrm{VH}) ; 12$ - horizontal hodoscope $(\mathrm{HH}) ; 13$ - aerogel Cherenkov (ChA); 14 - heavy gas Cherenkov (ChF); 15 - nitrogen Cherenkov (ChN); 16 preshower (PSh); 17 - muon detector $(\mathrm{Mu})$. 
a continuum background from free (unbound) "Coulomb pairs" produced from short lived sources $(\rho, \Delta, \ldots)$. Other background sources are "nonCoulomb pairs", where one or both pions originate from a long-lived source $\left(\eta, \eta^{\prime}, \Lambda, \ldots\right)$ and accidental coincidences from different proton-nucleus interactions.

\section{Study of $\pi^{+} \pi^{-}$atoms}

Study of $\pi^{+} \pi^{-}$atoms in the DIRAC experiment was fulfilled in collisions of 20 and $24 \mathrm{GeV} / c$ protons, delivered by the CERN PS, hitting a thin nickel foil target of 94 or $98 \mu \mathrm{m}$ thickness for different run periods [10]. Figure 2 shows observation of $\pi^{+} \pi^{-}$"atomic pairs" over the background of free pairs. Totally in all data samples, there were observed more than 21000 "atomic pairs".
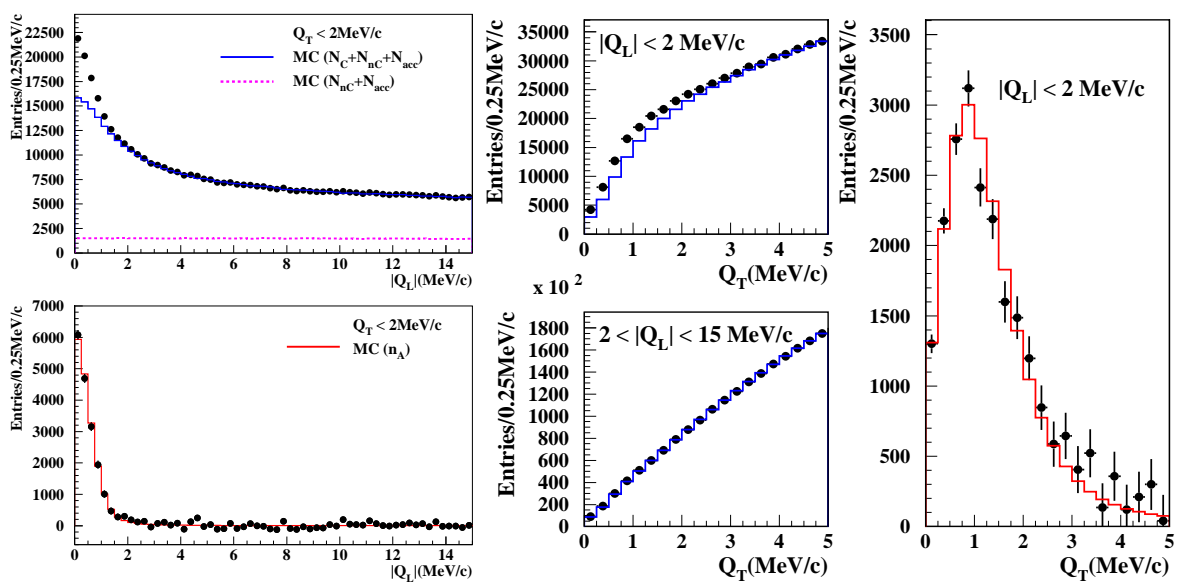

Fig. 2. Left: Fit of longitudinal projections $\left|Q_{\mathrm{L}}\right|$ of the $\pi^{+} \pi^{-}$spectrum from data (dots) and simulation (MC lines). The top plot shows the experimental spectrum compared with the simulated background components (no pionium signal), with (solid line) and without (dotted line) Coulomb pairs $\left(N_{\mathrm{C}}\right)$. The bottom plot shows the experimental $\left|Q_{\mathrm{L}}\right|$ spectrum after background subtraction and the simulated pionium spectrum. Centre: Fit of transverse projections $Q_{\mathrm{T}}$ of the $\pi^{+} \pi^{-}$spectrum from data (dots) and simulation (line). The plots correspond to different $Q_{\mathrm{L}}$ regions: top plot in the $A_{2 \pi}$ signal region (low $\left|Q_{\mathrm{L}}\right|$ ) and bottom left plot away from it (higher $\left|Q_{\mathrm{L}}\right|$ ). The right plot shows the $Q_{\mathrm{T}}$ spectrum after background subtraction and the simulated pionium spectrum.

The final measurement of the ground-state $A_{2 \pi}$ lifetime yields $\tau=$ $\left(\left.\left.3.15_{-0.19}^{+0.20}\right|_{\text {stat }}{ }_{-0.18}^{+0.20}\right|_{\text {syst }}\right) \times 10^{-15} \mathrm{~s}$. This value is in agreement with $\mathrm{ChPT}$ prediction $\tau=(2.9 \pm 0.1) \times 10^{-15} \mathrm{~s}[6]$. The measured lifetime value corre- 
sponds to the $\pi \pi$ scattering length difference

$$
\left|a_{0}-a_{2}\right|=\left(\left.\left.0.2533_{-0.0078}^{+0.0080}\right|_{\text {stat }}{ }_{-0.0073}^{+0.0078}\right|_{\text {syst }}\right) m_{\pi^{+}}^{-1} .
$$

\section{Observation of $\pi^{ \pm} K^{\mp}$ atoms}

$\pi^{ \pm} K^{\mp}$ atoms were detected in measurements with three targets: a platinum foil of $25.7 \mu \mathrm{m}$ thick and a nickel foil of 98 or $108 \mu \mathrm{m}$ for different run periods [11-13]. Figures 3 present experimental data on observation of $\pi K$ atomic pairs of both sign for 1-dimensional analysis over $Q$. Table I summarizes a number of observed $\pi K$ atomic pairs for all types of analyses.
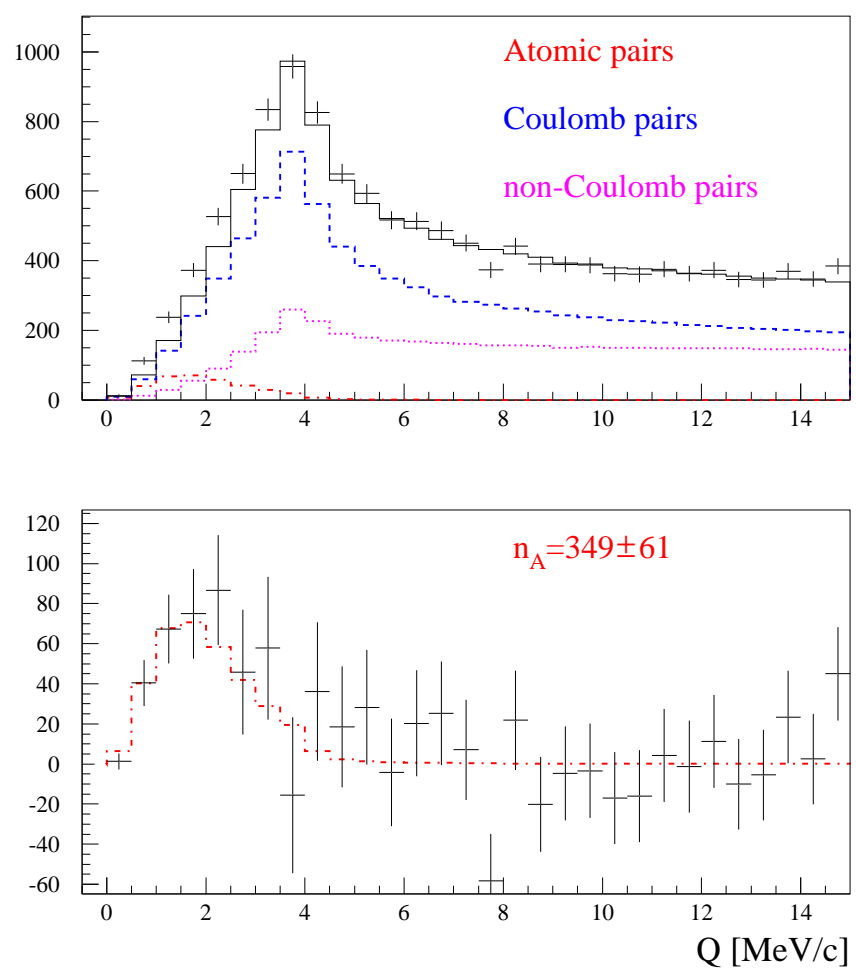

Fig. 3. (Color online) $Q$ distribution of experimental $\pi^{-} K^{+}$and $\pi^{+} K^{-}$pairs fitted by the sum of simulated distributions of atomic (dot-dashed/red), Coulomb (dashed/blue) and non-Coulomb pairs (dotted/magenta). Free (Coulomb and nonCoulomb) pairs are shown as solid/black line. Bottom: Difference distribution between experimental and simulated free pair distributions compared with simulated atomic pairs. The number of observed atomic pairs is denoted by $n_{\mathrm{A}}$. 
TABLE I

Atomic pair numbers $n_{\mathrm{A}}$ by analysing over full relative momentum $Q$ and its longitudinal $Q_{\mathrm{L}}$ and transverse $Q_{\mathrm{T}}$ components: the 1-dimensional of $Q$ and $\left|Q_{\mathrm{L}}\right|$ distributions and the 2-dimensional of $\left(\left|Q_{\mathrm{L}}\right|, Q_{\mathrm{T}}\right)$ distribution. Only statistical errors are given.

\begin{tabular}{c|c|c|c}
\hline \hline Analysis & $\pi^{-} K^{+}$ & $\pi^{+} K^{-}$ & $\pi^{-} K^{+}$and $\pi^{+} K^{-}$ \\
\hline$Q$ & $243 \pm 51(4.7 \sigma)$ & $106 \pm 32(3.3 \sigma)$ & $349 \pm 61(5.7 \sigma)$ \\
$\left|Q_{\mathrm{L}}\right|$ & $164 \pm 79(2.1 \sigma)$ & $67 \pm 47(1.4 \sigma)$ & $230 \pm 92(2.5 \sigma)$ \\
$\left|Q_{\mathrm{L}}\right|, Q_{\mathrm{T}}$ & $237 \pm 50(4.7 \sigma)$ & $78 \pm 32(2.5 \sigma)$ & $314 \pm 59(5.3 \sigma)$
\end{tabular}

The final result for $\pi K$ atom lifetime, using all the statistics of the $\mathrm{Ni}$ and $\mathrm{Pt}$ data samples, is $\tau=\left(\left.5.5_{-2.8}^{+5.0}\right|_{\text {tot }}\right) \times 10^{-15} \mathrm{~s}$. This value of lifetime corresponds to the following value of the $\pi K$ scattering lengths combination: $\frac{1}{3}\left|a_{1 / 2}-a_{3 / 2}\right|=\left(\left.0.072_{-0.020}^{+0.031}\right|_{\text {tot }}\right) M_{\pi}^{-1}$.

\section{Observation of long-lived states of $\pi^{+} \pi^{-}$atoms}

During the last stage of data taking, DIRAC had as objective the observation of long-lived states of $\pi^{+} \pi^{-}$atoms [14]. The long-lived states are the states with non-zero orbital quantum numbers $(l>0)$, for which the strong interaction is suppressed. As a result, the lifetime and mean path of such states are a few order of magnitude higher compared to the ground state. For example, at $\gamma=16$, the mean paths of $2 p$ and $3 p$ states are $5.7 \mathrm{~cm}$ and $19 \mathrm{~cm}$ correspondingly, compared to $0.02 \mathrm{~mm}$ for the ground state. The long-lived states are produced at excitation of atoms, primary produced only in states with zero orbital quantum numbers ( $s$-states). To observe the objects with such macroscopic path, a platinum foil of $2 \mu \mathrm{m}$ was installed at the distance of $10 \mathrm{~cm}$ downstream of the primary beryllium target of $100 \mu \mathrm{m}$. The platinum foil was placed above the proton beam to exclude interactions. A large fraction of the long-lived states, generated in $\mathrm{Be}$, reaches the $\mathrm{Pt}$ foil and breaks up, thus providing an extra "atomic pairs". For significant suppression of the background of $\pi^{+} \pi^{-}$pairs produced in Be foil, a permanent magnet with horizontal field of $0.02 \mathrm{Tm}$ bending power was placed between the foils (see Fig. 4). Calculations show that about $7 \%$ of produced atoms leave the target in excited long-lived states and $4 \%$ reach the $\mathrm{Pt}$ foil, where about $90 \%$ of them break up. There were detected $436 \pm\left. 57\right|_{\text {stat }} \pm\left. 23\right|_{\text {syst }}=436 \pm\left. 61\right|_{\text {tot }}$ of such pairs. This result corresponds to a $7.1 \sigma$ effect. 


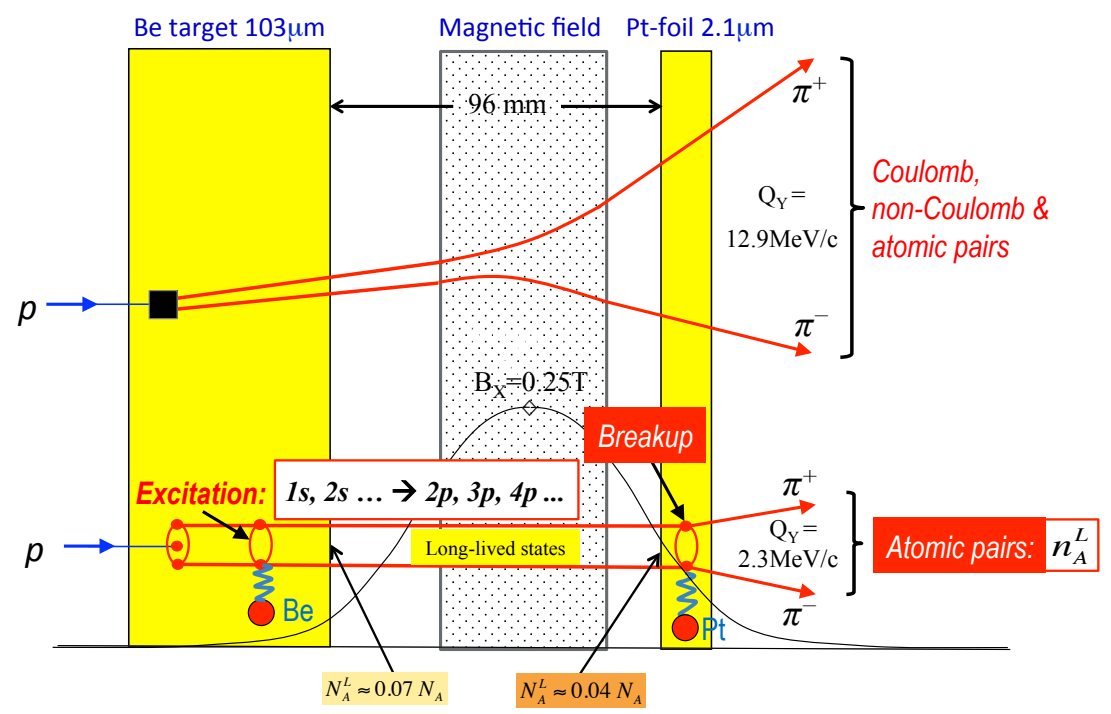

Fig. 4. Method to observe long-lived $\pi^{+} \pi^{-}$atoms by means of a breakup foil (Pt).

\section{REFERENCES}

[1] J. Uretsky, J. Palfrey, Phys. Rev. 121, 1798 (1961).

[2] J. Gasser et al., Phys. Rev. D 64, 016008 (2001).

[3] J. Gasser et al., Phys. Rep. 456, 167 (2008).

[4] J. Schweizer, Phys. Lett. B 587, 33 (2004).

[5] S. Weinberg, Physica A 96, 327 (1979); J. Gasser, H. Leutwyler, Phys. Lett. B 125, 325 (1983); Nucl. Phys. B 250, 465 (1985); 250, 517 (1985); 250, 539 (1985).

[6] G. Colangelo et al., Nucl. Phys. B 603, 125 (2001).

[7] Z. Fu, Phys. Rev. D 87, 074501 (2013); K. Sasaki et al., Phys. Rev. D 89, 054502 (2014); S.R. Beane et al., Phys. Rev. D 77, 014505 (2008).

[8] L.G. Afanasyev, A.V. Tarasov, Phys. At. Nucl. 59, 2130 (1996); C. Santamarina et al., J. Phys. B 36, 4273 (2003); L. Afanasyev et al., J. Phys. G 25, B7 (1999); L. Afanasyev et al., Phys. Rev. D 65, 096001 (2002).

[9] B. Adeva et al., Nucl. Instrum. Methods Phys. Res. A 515, 467 (2003); 839, 52 (2016).

[10] B. Adeva et al., Phys. Lett. B 704, 24 (2011).

[11] B. Adeva et al., Phys. Lett. B 674, 11 (2009).

[12] B. Adeva et al., Phys. Lett. B 735, 288 (2014).

[13] B. Adeva et al., Phys. Rev. Lett. 117, 112001 (2016).

[14] B. Adeva et al., Phys. Lett. B 751, 12 (2015). 